\title{
Depth Wise Distribution of Primary Nutrients in Pear Orchard Soils of Kashmir, India
}

\author{
M.A. Dar ${ }^{1}$, Rehana Rasool ${ }^{1}$, Masrat Maqbool ${ }^{1 *}$, J.A. Wani ${ }^{1}$, M.Y. Bhat ${ }^{2}$ and \\ Shazia Ramzan ${ }^{1}$
}

\author{
${ }^{1}$ Division of Soil Science, ${ }^{2}$ Division of Pomology, Sheri-Kashmir University of Agricultural \\ Sciences and Technology of Kashmir, Shalimar J\&K 190015, India \\ *Corresponding author
}

\section{A B S T R A C T}

\section{Keywords \\ Altitude, Primary nutrient, Soils, Pear, Kashmir. \\ Article Info \\ Accepted: \\ 29 May 2017 \\ Available Online: \\ 10 June 2017}

The present study revealed that $\mathrm{pH}$ of pear orchard soils increased with decrease in altitude while as organic carbon decreased with decrease in altitude. The textural class of soils ranged between clay loam to silt clay loam with normal electrical conductivity. The available nitrogen status was low to medium, whereas, available phosphorus status was medium to high and available potassium status was high. The effect of altitude on the available nitrogen, phosphorus and potassium was found significant statistically, thereby showing that altitude bears effect on their content in pear orchard soils of Kashmir.

\section{Introduction}

Fruit production is considered as the backbone of the economy of Jammu And Kashmir State. About 80 percent of the rural population is directly as well as indirectly related with this occupation. The fruit production in the state is 5515.41 thousand metric tones during 2006-07 from an area of 472.70 thousand hectares. Among temperate fresh fruits grown in the state, the pear is rank next to apple. It is cultivated in valley of Kashmir and higher reaches of Jammu like Doda, Poonch and Rajouri. The area under pear is 15.99 thousand hectares with a production of 70.84 thousand metric tones (Anonymous, 2007). Nutrition plays an important role in the production of quality fruits. In pear also, proper amount of nutrients is essential for the growth and development of plants leading to maximum production of quality fruits. It has been observed that availability of nutrients to the plants is affected by many factors, which includes, soil texture, moisture, soil $\mathrm{pH}$, slope, drainage, aspect, altitude, climate, stock, scion, etc. The effect of altitude on availability of plant nutrients is obvious as it affects the various factors of climate like temperature, rainfall, solar radiation etc. These in turn bear effect on the availability of nutrients to the plants. The altitude of valley varied from 1500 to 2500 meters above mean sea level. The present investigation has been taken to study the effect of altitude on the availability of primary nutrients like nitrogen, phosphorus 
and potassium in pear orchard soils of Kashmir valley as no such investigation/research was conducted so far with respect to pear orchard soils.

\section{Materials and Methods}

\section{Description and collection of soil samples from experimental site}

The study are i.e. Kashmir valley is spread over Latitude of $32^{\circ} 17^{\prime}$ to $80^{\circ} 30^{\prime} \mathrm{E}$. The present investigation was carried out in 21 pear orchards of uniform age group (15-20 years) from pear growing areas of the valley located at different altitudes and grouped into three groups i.e., high altitude, mid altitude and low altitude as shown in table 1. Seven sites from each altitude were selected which represented the different districts (Budgam, Kulgam, Pulwama, Ganderbal, Kupwara and Srinagar) of the valley. The texture of the soils ranged from clay loam to silty clay loam. Soil samples were collected up to depth of 50 $\mathrm{cm}$ with an increment of $25 \mathrm{~cm}$. The samples were air dried and crushed with a wooden pestle and mortar followed by sieving through a sieve of $2 \mathrm{~mm}$ size and stored in polythene bags for analysis. A portion was passed through a sieve of $0.5 \mathrm{~mm}$ and stored separately for estimation of organic carbon.

\section{Methods for estimation of various soil} parameters

The $\mathrm{pH}$ and electrical conductivity were estimated in 1:2.5 soil water suspensions by standard procedure given by Jackson (1973). The organic carbon was determined by the method outlined by Walkley and Black (1934) and calcium carbonate was found by the method given by Piper (1966). The available nitrogen was determined by alkaline permanganate method outlined by Subbiah and Asija (1956). Available phosphorus was extracted with $0.5 \mathrm{~N}$ sodium bicarbonate at $\mathrm{pH}$
8.5 (Olsen et al., 1954) and estimated by ammonium molybdate method (Jackson, 1973). The available potassium was extracted with normal ammonium acetate and determined on flame photometer as described by Jackson (1973).

\section{Experimental design}

Mean values were used to obtain estimation of variance components as per methods suggested by Panse and Sukhatime (1967). Nutrient standards as per established procedure (Table 2) were used for the determination of nutrient status in soils.

\section{Results and Discussion}

\section{Depth-wise distribution of physico-} chemical attributes

\section{Soil pH (1:2.5)}

Soil reaction being the most important physico-chemical property influences the soil health from chemical, physical, biological and mineralogical point of view. The $\mathrm{pH}$ in the surface layer $(0-25 \mathrm{~cm})$ ranged from 6.10 to $6.41,6.52$ to 6.75 and 6.92 to 7.76 in high, mid and low altitudes, respectively, with an overall average and mean value of 6.10 to 7.76 and 6.75 in surface soil respectively (Table 3). The soil $\mathrm{pH}$ in subsurface layer $(25-50 \mathrm{~cm})$ ranged from $6.20-6.48,6.60-6.83$ and 6.99-7.85 in high, mid and low altitudes, respectively, with an overall range and mean value of 6.20 to 7.85 and 6.87 in subsurface soil respectively. The $\mathrm{pH}$ decreases with increase in altitude, but increases with increase in soil depth, in general soils were slightly acidic to slightly alkaline. However the effect of altitude on soil $\mathrm{pH}$ was observed to be significant. This could be due to leaching of bases due to high rainfall, besides higher amount of organic matter in high altitude soils. These are supported with the 
findings of Lahiri and Chakravarti (1989) and Mandal et al., (1990).

\section{Electrical conductivity $\left(\mathrm{dSm}^{-1}\right)$}

Soils generally differ in their salt content, which affects their ability to grow crops. Excess salts interfere with water and nutrient uptake. The soil Ec in the surface layer (0$25 \mathrm{~cm}$ ) varied from 0.10 to $0.25,0.14$ to 0.42 and 0.16 to $0.44 \mathrm{dSm}^{-1}$ in high, mid and low altitudes, respectively, with an overall range and mean value of 0.10 to 0.44 and $0.26 \mathrm{dSm}^{-1}$ in surface soil respectively (Table 4). Whereas, in subsurface layer $(25-50 \mathrm{~cm})$ it ranged from 0.13 to $0.23,0.18$ to 0.34 and 0.11 to $0.37 \mathrm{dSm}^{-1}$ in high, mid and low altitudes, respectively, with an overall range and mean value of 0.13 to 0.37 and $0.21 \mathrm{dSm}^{-}$ 1 in subsurface soil respectively. The electrical conductivity of all soils was observed normal and it showed an irregular trend with an increase in soil depth (Table 4). The electrical conductivity varied significantly in these soils with relatively lower value in soils of high altitude. This could be due to leaching of soluble salts and runoff transportation due to high precipitation. This is in accordance with the results of Balanagoudar and Satyanarayana (1990) and Najar (2002).

\section{Calcium carbonate (\%)}

Calcium in soils effects the soil reaction, thereby affecting the availability of plant nutrients. The calcium carbonate in the surface layer $(0-25 \mathrm{~cm})$ varied from 6.4 to 8.2 , 8.4 to 9.8 and 6.8 to 8.2 percent, in high, mid and low altitudes, respectively, with an overall range and mean value of 6.4 to 9.8 percent and 7.9 percent in surface soil respectively (Table 5). Whereas, in subsurface layer $(25-50 \mathrm{~cm})$ it ranged from 7.0 to $9.0,8.8$ to 10.2 and 7.2 to 8.6 percent in high, mid and low altitudes, respectively, With an overall range and mean value of 7.0 to 10.2 percent and 8.39 percent in subsurface soil respectively with higher content in mid altitude soils, which may be due to calcareous nature of parent material. In general, the contents of calcium carbonate increased with depth, which could be due to leaching of bases from surface to subsurface layers. Similar results were reported by Talib (1984) and Kher and Singh (1993).

\section{Organic carbon content (\%)}

Soil organic carbon serves as an index of soil productivity. The organic carbon in the surface layer $(0-25 \mathrm{~cm})$ varied from 1.28 to 2.36, 1.14 to 2.21 and 0.66 to 1.72 percent, in high, mid and low altitudes, respectively, with an overall range and mean value of 0.66 to 2.36 percent and 1.54 percent in surface soil respectively (Table 6). Whereas, in subsurface layer $(25-50 \mathrm{~cm})$ organic carbon ranged from 1.02 to $1.56,0.99$ to 1.49 and 0.54 to 1.56 percent in high, mid and low altitudes, respectively, With an overall range and mean value of 0.54 to 1.56 percent and 1.10 percent in subsurface soil respectively. The surface soils showed higher content of organic carbon, which decreases with an increase in soil depth. The high content of organic carbon in surface layer is due to natural vegetation and addition of organic matter to pear orchards. The organic carbon content varied significantly with altitude and high content of organic carbon was observed in high altitude soils and in general decreases with the decrease in altitude. This is due to luxurious vegetation because of high rainfall and slow rate of decomposition due to low temperature leading to accumulation of organic matter at high altitudes. These findings are supported by Minhas and Bora (1982) and Sharma et al., (2005). 
Table.1 Selected sites with altitudes of pear orchards

$\begin{array}{llll}\text { S.No. } & \text { Location } & \text { District } & \begin{array}{l}\text { Altitude } \\ \text { (a.m.s.l) } \\ \text { (meters) }\end{array} \\ 1 & \text { Chrarisharief } & \text { Budgam } & 2100 \\ 2 & \text { Pakherpora } & \text { Budgam } & 2020 \\ 3 & \text { Footlipora } & \text { Budgam } & 2000 \\ 4 & \text { Kamrazipora } & \text { Pulwama } & 1920 \\ 5 & \text { Kanigam } & \text { Pulwama } & 1880 \\ 6 & \text { Tujan } & \text { Pulwama } & 1850 \\ 7 & \text { Adijan } & \text { Kulgam } & 1840 \\ 8 & \text { Drabagam } & \text { Pulwama } & 1800 \\ 9 & \text { Pombay } & \text { Kulgam } & 1800 \\ 10 & \text { Astanpora } & \text { Srinagar } & 1790 \\ 11 & \text { Rajpora } & \text { Pulwama } & 1780 \\ 12 & \text { Tral } & \text { Pulwama } & 1760 \\ 13 & \text { Nawpora } & \text { Budgam } & 1750 \\ 14 & \text { Pirpora } & \text { Pulwama } & 1730 \\ 15 & \text { Wakura } & \text { Ganderbal } & 1690 \\ 16 & \text { Zazna } & \text { Ganderbal } & 1640 \\ 17 & \text { New Thead } & \text { Srinagar } & 1620 \\ 18 & \text { Manzgam } & \text { Srinagar } & 1580 \\ 19 & \text { Khanda } & \text { Budgam } & 1580 \\ 20 & \text { Handwara } & \text { Kupwara } & 1560 \\ 21 & \text { Pohru } & \text { Budgam } & 1520\end{array}$

Table.2 Critical limits of available nutrient elements in soils

\begin{tabular}{lcclll}
$\begin{array}{l}\text { Nutrient } \\
\text { Element }\end{array}$ & Unit & Low & Medium & High & Reference \\
$\begin{array}{l}\text { Organic } \\
\text { carbon }\end{array}$ & $\%$ & $<0.5$ & $0.5-1.0$ & $>1.0$ & Walkley and Black (1934) \\
$\begin{array}{l}\text { Nitrogen } \\
\text { Phosphorus }\end{array}$ & $\mathrm{ppm}$ & $<125$ & $125-250$ & $>250$ & Subbiah and Asija (1956) \\
Potassium & $\mathrm{ppm}$ & $<4$ & $4-11$ & $>11$ & Olsen et al., (1954) \\
Sulphur & $\mathrm{ppm}$ & $<10$ & $44-125$ & $>125$ & Hanway and Heidal (1952) \\
Zinc & $\mathrm{ppm}$ & $<0.6$ & $0.6-1.2$ & $>1.2$ & Tanwar and Mohan (1964) \\
Copper & $\mathrm{ppm}$ & $<0.2$ & $0.2-2.0$ & $>2.0$ & Follet and Mann (1975) \\
Manganese & $\mathrm{ppm}$ & $<1.0$ & - & - & Follet and Lindsay (1970) \\
Iron & $\mathrm{ppm}$ & $<4.5$ & - & - & Lindsay and Norvell (1978) \\
\hline & & & & & \\
\hline
\end{tabular}


Table.3 Soil reaction $(\mathrm{pH})$ in surface and sub-surface soils with different altitudes of pear orchard

\begin{tabular}{|c|c|c|c|c|}
\hline Altitude Range & Location & $\begin{array}{l}\text { Altitude } \\
\text { (meters) }\end{array}$ & \multicolumn{2}{|c|}{$\begin{array}{c}\text { Depth } \\
\text { (cm) }\end{array}$} \\
\hline \multirow[t]{25}{*}{ High Altitude } & & & $0-25$ & $25-50$ \\
\hline & Chrarisharief & 2100 & 6.10 & 6.20 \\
\hline & Pakherpora & 2020 & 6.26 & 6.37 \\
\hline & Eootlipora & 2000 & 6.28 & 6.39 \\
\hline & Kamrazipora & 1920 & 6.35 & 6.41 \\
\hline & Kanigam & 1880 & 6.39 & 6.42 \\
\hline & Tujan & 1850 & 6.41 & 6.47 \\
\hline & Adijan & 1840 & 6.40 & 6.48 \\
\hline & \multicolumn{4}{|c|}{$\begin{array}{c}\text { Surface Range: 6.10-6.41 } \\
\text { Mean: } 6.31 \\
\text { Sub-surface Range: } 6.20-6.48 \\
\text { Mean: } 6.39\end{array}$} \\
\hline & Drabagam & 1800 & 6.52 & 6.60 \\
\hline & Pombay & 1800 & 6.61 & 6.67 \\
\hline & Asthanpora & 1790 & 6.54 & 6.63 \\
\hline & Rajpora & 1780 & 6.65 & 6.74 \\
\hline & Tral & 1760 & 6.69 & 6.87 \\
\hline & Nawpora & 1750 & 6.70 & 6.76 \\
\hline & Pirpora & 1730 & 6.75 & 6.83 \\
\hline & \multicolumn{4}{|c|}{$\begin{array}{c}\text { Surface Range: } 6.52-6.75 \\
\text { Mean: } 6.64 \\
\text { Sub-surface Range: } 6.60-6.83 \\
\text { Mean: } 6.73\end{array}$} \\
\hline & Wakura & 1690 & 6.92 & 6.99 \\
\hline & Zazan & 1640 & 7.20 & 7.38 \\
\hline & New Thead & 1620 & 7.32 & 7.35 \\
\hline & Manzgam & 1580 & 7.36 & 7.45 \\
\hline & Khanda & 1580 & 6.94 & 7.65 \\
\hline & Handwara & 1560 & 7.62 & 7.71 \\
\hline & Pohru & 1520 & 7.76 & 7.85 \\
\hline & \multicolumn{4}{|c|}{$\begin{array}{c}\text { Surface Range: 6.92-7.76 } \\
\text { Mean: 7.30 } \\
\text { Sub-surface Range: } 6.99-7.85 \\
\text { Mean: } 7.48\end{array}$} \\
\hline $\operatorname{LSD}(0.05)$ & \multicolumn{4}{|c|}{0.14} \\
\hline \pm SED & \multicolumn{4}{|c|}{0.07} \\
\hline
\end{tabular}


Table.4 Electrical conductivity in surface and sub-surface soils with different altitudes of pear orchard

\begin{tabular}{|c|c|c|c|c|}
\hline \multirow{2}{*}{$\begin{array}{c}\text { Altitude Range } \\
\text { High Altitude }\end{array}$} & \multirow[t]{2}{*}{ Location } & \multirow{2}{*}{$\begin{array}{c}\text { Altitude } \\
\text { (meters) } \\
\text { (amsl) } \\
\end{array}$} & \multicolumn{2}{|c|}{$\begin{array}{c}\text { Depth } \\
\text { (cm) }\end{array}$} \\
\hline & & & $0-25$ & $25-50$ \\
\hline & Chrarisharief & 2100 & 0.10 & 0.16 \\
\hline & Pakherpora & 2020 & 0.16 & 0.19 \\
\hline & Eootlipora & 2000 & 0.25 & 0.13 \\
\hline & Kamrazipora & 1920 & 0.12 & 0.17 \\
\hline & Kanigam & 1880 & 0.24 & 0.18 \\
\hline & Tujan & 1850 & 0.15 & 0.23 \\
\hline & Adijan & 2100 & 0.10 & 0.16 \\
\hline & \multicolumn{4}{|c|}{$\begin{array}{c}\text { Surface Range: } 0.10-0.25 \\
\text { Mean: } 0.18 \\
\text { Sub-surface Range: } 0.13-0.23 \\
\text { Mean: } 0.17\end{array}$} \\
\hline & Drabagam & 1800 & 0.15 & 0.22 \\
\hline & Pombay & 1800 & 0.35 & 0.26 \\
\hline & Asthanpora & 1790 & 0.14 & 0.23 \\
\hline & Rajpora & 1780 & 0.42 & 0.25 \\
\hline & Tral & 1760 & 0.37 & 0.34 \\
\hline & Nawpora & 1750 & 0.25 & 0.18 \\
\hline & Pirpora & 1730 & 0.35 & 0.19 \\
\hline & \multicolumn{4}{|c|}{$\begin{array}{c}\text { Surface Range: } 0.14-0.42 \\
\text { Mean: } 0.29 \\
\text { Sub-surface Range: } 0.18-0.34 \\
\text { Mean: } 0.23\end{array}$} \\
\hline & Wakura & 1690 & 0.27 & 0.11 \\
\hline & Zazan & 1640 & 0.31 & 0.37 \\
\hline & New Thead & 1620 & 0.16 & 0.28 \\
\hline & Manzgam & 1580 & 0.44 & 0.28 \\
\hline & Khanda & 1580 & 0.24 & 0.18 \\
\hline & Handwara & 1560 & 0.36 & 0.25 \\
\hline & Pohru & 1520 & 0.29 & 0.17 \\
\hline & \multicolumn{4}{|c|}{$\begin{array}{c}\text { Surface Range: } 0.16-0.44 \\
\text { Mean: } 0.30 \\
\text { Sub-surface Range: } 0.11-0.37 \\
\text { Mean: } 0.23\end{array}$} \\
\hline LSD(0.05) & \multicolumn{4}{|c|}{0.06} \\
\hline 土SED & \multicolumn{4}{|c|}{$\mathbf{0 . 0 3}$} \\
\hline
\end{tabular}


Table.5 Calcium carbonate (\%) in surface and sub-surface soils with different altitudes of pear orchard

\begin{tabular}{|c|c|c|c|c|}
\hline Altitude Range & Location & $\begin{array}{l}\text { Altitude } \\
\text { (meters) }\end{array}$ & \multicolumn{2}{|c|}{$\begin{array}{c}\text { Depth } \\
\text { (cm) }\end{array}$} \\
\hline \multirow[t]{25}{*}{ High Altitude } & & & $0-25$ & $25-50$ \\
\hline & Chrarisharief & 2100 & 6.4 & 7.0 \\
\hline & Pakherpora & 2020 & 6.6 & 7.4 \\
\hline & Eootlipora & 2000 & 6.6 & 7.6 \\
\hline & Kamrazipora & 1920 & 7.4 & 7.6 \\
\hline & Kanigam & 1880 & 7.6 & 7.8 \\
\hline & Tujan & 1850 & 8.0 & 8.4 \\
\hline & Adijan & 1840 & 8.2 & 9.0 \\
\hline & \multicolumn{4}{|c|}{$\begin{array}{c}\text { Surface Range: } 6.4-8.2 \\
\text { Mean: } 7.26 \\
\text { Sub-surface Range: } 7.0-9.0 \\
\text { Mean: } 7.82\end{array}$} \\
\hline & Drabagam & 1800 & 8.4 & 8.8 \\
\hline & Pombay & 1800 & 8.6 & 9.4 \\
\hline & Asthanpora & 1790 & 8.4 & 9.0 \\
\hline & Rajpora & 1780 & 8.8 & 9.2 \\
\hline & Tral & 1760 & 9.6 & 10.2 \\
\hline & Nawpora & 1750 & 9.6 & 10.2 \\
\hline & Pirpora & 1730 & 9.8 & 10.0 \\
\hline & \multicolumn{4}{|c|}{$\begin{array}{c}\text { Surface Range: } 8.4-9.8 \\
\text { Mean: } 9.03 \\
\text { Sub-surface Range: } 8.8-10.2 \\
\text { Mean: } 9.54\end{array}$} \\
\hline & Wakura & 1690 & 6.8 & 7.2 \\
\hline & Zazan & 1640 & 7.0 & 7.7 \\
\hline & New Thead & 1620 & 7.0 & 7.4 \\
\hline & Manzgam & 1580 & 7.4 & 7.6 \\
\hline & Khanda & 1580 & 7.6 & 7.8 \\
\hline & Handwara & 1560 & 7.8 & 8.4 \\
\hline & Pohru & 1520 & 8.2 & 8.6 \\
\hline & \multicolumn{4}{|c|}{$\begin{array}{c}\text { Surface Range: } 6.8-8.2 \\
\text { Mean: } 7.40 \\
\text { Sub-surface Range: } 7.2-8.6 \\
\text { Mean: } 7.81\end{array}$} \\
\hline LSD(0.05) & \multicolumn{4}{|c|}{0.45} \\
\hline \pm SED & \multicolumn{4}{|c|}{0.22} \\
\hline
\end{tabular}


Table.6 Organic carbon (\%) in surface and sub-surface soils with different altitudes of pear orchard

\begin{tabular}{|c|c|c|c|c|}
\hline Altitude Range & Location & $\begin{array}{l}\text { Altitude } \\
\text { (meters) }\end{array}$ & \multicolumn{2}{|c|}{$\begin{array}{c}\text { Depth } \\
(\mathbf{c m})\end{array}$} \\
\hline \multirow[t]{25}{*}{ High Altitude } & & & $0-25$ & 25- 50 \\
\hline & Chrarisharief & 2100 & 2.36 & 1.53 \\
\hline & Pakherpora & 2020 & 1.95 & 1.56 \\
\hline & Eootlipora & 2000 & 2.04 & 1.11 \\
\hline & Kamrazipora & 1920 & 1.87 & 1.19 \\
\hline & Kanigam & 1880 & 1.32 & 1.17 \\
\hline & Tujan & 1850 & 1.65 & 1.17 \\
\hline & Adijan & 1840 & 1.28 & 1.02 \\
\hline & \multicolumn{4}{|c|}{$\begin{array}{c}\text { Surface Range: 1.28-2.36 } \\
\text { Mean: 1.78 } \\
\text { Sub-surface Range: } 1.02-1.56 \\
\text { Mean: } 1.25\end{array}$} \\
\hline & Drabagam & 1800 & 1.78 & 1.17 \\
\hline & Pombay & 1800 & 1.27 & 1.15 \\
\hline & Asthanpora & 1790 & 2.21 & 1.49 \\
\hline & Rajpora & 1780 & 1.82 & 1.27 \\
\hline & Tral & 1760 & 1.45 & 1.07 \\
\hline & Nawpora & 1750 & 1.14 & 0.99 \\
\hline & Pirpora & 1730 & 1.52 & 1.03 \\
\hline & \multicolumn{4}{|c|}{$\begin{array}{c}\text { Surface Range: } 1.14-2.21 \\
\text { Mean: } 1.60 \\
\text { Sub-surface Range: } 0.99-1.49 \\
\text { Mean: } 1.17\end{array}$} \\
\hline & Wakura & 1690 & 1.61 & 1.02 \\
\hline & Zazan & 1640 & 1.09 & 0.99 \\
\hline & New Thead & 1620 & 1.46 & 1.00 \\
\hline & Manzgam & 1580 & 1.19 & 0.87 \\
\hline & Khanda & 1580 & 1.72 & 1.21 \\
\hline & Handwara & 1560 & 0.92 & 0.54 \\
\hline & Pohru & 1520 & 0.66 & 0.58 \\
\hline & \multicolumn{4}{|c|}{$\begin{array}{c}\text { Surface Range: 0.66-1.72 } \\
\text { Mean: 1.24 } \\
\text { Sub-surface Range: } 0.54-1.21 \\
\text { Mean: 0.89 }\end{array}$} \\
\hline $\operatorname{LSD}(0.05)$ & \multicolumn{4}{|c|}{0.22} \\
\hline 土SED & \multicolumn{4}{|c|}{0.11} \\
\hline
\end{tabular}


Table.7 Nitrogen $\left(\mathrm{Kg} / \mathrm{ha}^{-1}\right)$ in surface and sub-surface soils with different altitudes of pear orchard

\begin{tabular}{|c|c|c|c|c|}
\hline Altitude Range & Location & $\begin{array}{l}\text { Altitude } \\
\text { (meters) }\end{array}$ & \multicolumn{2}{|c|}{$\begin{array}{c}\text { Depth } \\
\text { (cm) }\end{array}$} \\
\hline \multirow[t]{25}{*}{ High Altitude } & & & $0-25$ & 25- 50 \\
\hline & Chrarisharief & 2100 & 439.4 & 400.0 \\
\hline & Pakherpora & 2020 & 426.9 & 342.2 \\
\hline & Eootlipora & 2000 & 408.5 & 316.2 \\
\hline & Kamrazipora & 1920 & 378.5 & 285.8 \\
\hline & Kanigam & 1880 & 395.5 & 303.3 \\
\hline & Tujan & 1850 & 377.2 & 316.2 \\
\hline & Adijan & 1840 & 384.3 & 309.1 \\
\hline & \multicolumn{4}{|c|}{$\begin{array}{c}\text { Surface Range: } 377.2-439.4 \\
\text { Mean: } 401.54 \\
\text { Sub-surface Range: } 285.8-400.0 \\
\text { Mean: } 324.73\end{array}$} \\
\hline & Drabagam & 1800 & 419.3 & 333.3 \\
\hline & Pombay & 1800 & 339.1 & 257.1 \\
\hline & Asthanpora & 1790 & 383.4 & 314.5 \\
\hline & Rajpora & 1780 & 325.2 & 228.9 \\
\hline & Tral & 1760 & 302.4 & 237.8 \\
\hline & Nawpora & 1750 & 283.5 & 242.3 \\
\hline & Pirpora & 1730 & 366.9 & 315.8 \\
\hline & \multicolumn{4}{|c|}{$\begin{array}{c}\text { Surface Range: } 283.5-419.3 \\
\text { Mean: 345.72 } \\
\text { Sub-surface Range: } 228.9-333.3 \\
\text { Mean: } 275.71\end{array}$} \\
\hline & Wakura & 1690 & 393.7 & 294.7 \\
\hline & Zazan & 1640 & 316.7 & 287.1 \\
\hline & New Thead & 1620 & 363.7 & 305.9 \\
\hline & Manzgam & 1580 & 272.3 & 164.8 \\
\hline & Khanda & 1580 & 360.1 & 280.4 \\
\hline & Handwara & 1560 & 272.3 & 172.9 \\
\hline & Pohru & 1520 & 207.8 & 177.4 \\
\hline & \multicolumn{4}{|c|}{$\begin{array}{c}\text { Surface Range: } 207.8-393.7 \\
\text { Mean: } 312.46 \\
\text { Sub-surface Range: } 164.8-305.9 \\
\text { Mean: } 240.51\end{array}$} \\
\hline LSD(0.05) & \multicolumn{4}{|c|}{32.61} \\
\hline \pm SED & \multicolumn{4}{|c|}{16.08} \\
\hline
\end{tabular}


Table.8 Phosphorus $\left(\mathrm{Kg} / \mathrm{ha}^{-1}\right)$ in surface and sub-surface soils with different altitudes of pear orchard

\begin{tabular}{|c|c|c|c|c|}
\hline Altitude Range & Location & $\begin{array}{l}\text { Altitude } \\
\text { (meters) }\end{array}$ & \multicolumn{2}{|c|}{$\begin{array}{l}\text { Depth } \\
\text { (cm) }\end{array}$} \\
\hline \multirow[t]{25}{*}{ High Altitude } & & & $0-25$ & 25- 50 \\
\hline & Chrarisharief & 2100 & 41.66 & 37.18 \\
\hline & Pakherpora & 2020 & 34.05 & 28.67 \\
\hline & Eootlipora & 2000 & 35.39 & 34.05 \\
\hline & Kamrazipora & 1920 & 38.98 & 27.78 \\
\hline & Kanigam & 1880 & 34.94 & 30.02 \\
\hline & Tujan & 1850 & 32.70 & 25.54 \\
\hline & Adijan & 1840 & 40.32 & 31.81 \\
\hline & \multicolumn{4}{|c|}{$\begin{array}{c}\text { Surface Range: } 32.70-41.66 \\
\text { Mean: } 36.87 \\
\text { Sub-surface Range: } 25.54-37.18 \\
\text { Mean: } 30.72\end{array}$} \\
\hline & Drabagam & 1800 & 40.77 & 34.05 \\
\hline & Pombay & 1800 & 37.63 & 32.70 \\
\hline & Asthanpora & 1790 & 39.42 & 36.74 \\
\hline & Rajpora & 1780 & 33.60 & 30.02 \\
\hline & Tral & 1760 & 33.15 & 26.43 \\
\hline & Nawpora & 1750 & 34.50 & 22.85 \\
\hline & Pirpora & 1730 & 33.15 & 25.09 \\
\hline & \multicolumn{4}{|c|}{$\begin{array}{c}\text { Surface Range: } 33.15-40.77 \\
\text { Mean: } 36.04 \\
\text { Sub-surface Range: } 22.85-36.74 \\
\text { Mean: } 29.70\end{array}$} \\
\hline & Wakura & 1690 & 39.42 & 24.19 \\
\hline & Zazan & 1640 & 29.57 & 28.67 \\
\hline & New Thead & 1620 & 33.60 & 27.78 \\
\hline & Manzgam & 1580 & 28.22 & 23.30 \\
\hline & Khanda & 1580 & 31.81 & 23.30 \\
\hline & Handwara & 1560 & 25.09 & 21.95 \\
\hline & Pohru & 1520 & 21.06 & 19.71 \\
\hline & \multicolumn{4}{|c|}{$\begin{array}{c}\text { Surface Range: } 21.06-39.42 \\
\text { Mean: } 29.81 \\
\text { Sub-surface Range: } 19.71-28.67 \\
\text { Mean: } 24.13\end{array}$} \\
\hline LSD(0.05) & \multicolumn{4}{|c|}{2.78} \\
\hline \pm SED & \multicolumn{4}{|c|}{1.37} \\
\hline
\end{tabular}


Table.9 Potassium $\left(\mathrm{Kg} / \mathrm{ha}^{-1}\right)$ in surface and sub-surface soils with different altitudes of pear orchard

\begin{tabular}{|c|c|c|c|c|}
\hline Altitude Range & Location & $\begin{array}{l}\text { Altitude } \\
\text { (meters) }\end{array}$ & \multicolumn{2}{|c|}{$\begin{array}{l}\text { Depth } \\
(\mathrm{cm})\end{array}$} \\
\hline \multirow[t]{25}{*}{ High Altitude } & & & $0-25$ & 25- 50 \\
\hline & Chrarisharief & 2100 & 456.9 & 389.7 \\
\hline & Pakherpora & 2020 & 407.6 & 407.6 \\
\hline & Eootlipora & 2000 & 474.8 & 347.2 \\
\hline & Kamrazipora & 1920 & 434.5 & 398.7 \\
\hline & Kanigam & 1880 & 356.1 & 331.5 \\
\hline & Tujan & 1850 & 416.6 & 277.7 \\
\hline & Adijan & 1840 & 371.8 & 315.8 \\
\hline & \multicolumn{4}{|c|}{$\begin{array}{c}\text { Surface Range: } 356.1-474.8 \\
\text { Mean: } 416.95 \\
\text { Sub-surface Range: } 277.7-407.6 \\
\text { Mean: } 352.64\end{array}$} \\
\hline & Drabagam & 1800 & 465.9 & 376.3 \\
\hline & Pombay & 1800 & 340.4 & 295.6 \\
\hline & Asthanpora & 1790 & 358.4 & 253.9 \\
\hline & Rajpora & 1780 & 416.6 & 383.0 \\
\hline & Tral & 1760 & 362.8 & 188.1 \\
\hline & Nawpora & 1750 & 331.5 & 284.4 \\
\hline & Pirpora & 1730 & 430.0 & 237.4 \\
\hline & \multicolumn{4}{|c|}{$\begin{array}{c}\text { Surface Range: } 331.5-465.9 \\
\text { Mean: } 386.56 \\
\text { Sub-surface Range: } 188.1-383.0 \\
\text { Mean: } 288.43\end{array}$} \\
\hline & Wakura & 1690 & 456.9 & 277.7 \\
\hline & Zazan & 1640 & 439.0 & 275.5 \\
\hline & New Thead & 1620 & 470.4 & 239.6 \\
\hline & Manzgam & 1580 & 403.2 & 206.0 \\
\hline & Khanda & 1580 & 374.0 & 188.1 \\
\hline & Handwara & 1560 & 349.4 & 228.4 \\
\hline & Pohru & 1520 & 318.0 & 235.2 \\
\hline & \multicolumn{4}{|c|}{$\begin{array}{c}\text { Surface Range: } 318.0-470.4 \\
\text { Mean: 401.61 } \\
\text { Sub-surface Range: } 188.1-277.7 \\
\text { Mean: } 235.84\end{array}$} \\
\hline LSD(0.05) & \multicolumn{4}{|c|}{31.38} \\
\hline \pm SED & \multicolumn{4}{|c|}{15.48} \\
\hline
\end{tabular}




\section{Depth-wise distribution of primary nutrients}

\section{Nitrogen $\left(\mathrm{Kg} / \mathrm{ha}^{-1}\right)$}

The available nitrogen in surface layers $(0-25$ $\mathrm{cm}$ ) of high, mid, and low altitude soils of pear orchards varied from 377.2 to 439.4, 283.5 to 419.3 and 207.8 to $393.7 \mathrm{Kg}$ per hectare with mean value of 401.5, 345.7 and $312.4 \mathrm{Kg}$ per hectare, respectively. While as in subsurface layer $(25-50 \mathrm{~cm})$ it ranged from 285.8 to $400.0,228.9$ to 333.3 and $164.8-305.9$ $\mathrm{Kg}$ per hectare with mean value of 324.73 , 275.71 and $240.51 \mathrm{Kg}$ per hectare, respectively (Table 7). It has been observed that 86 percent samples have medium status and 14 percent have low status of available nitrogen. This could be due to continuous removal of added as well as mineralized nitrogen by intensive cropping, leaching/ denitrification losses and also its correlation with organic matter content. The available nitrogen was high in surface layers with regular decreasing trend with soil depth. The available nitrogen differed significantly with altitude. Nitrogen was observed high in high altitude soils then in soils of mid and low altitude and it decreases with decrease in altitude, which may be due to climatic and physiographic conditions favourable for the accumulation of high organic matter content in high altitude. This is supported by the similar observations of Kaistha et al., (1990) and Najar et al., (2006).

\section{Phosphorus (Kg/ha $\left.{ }^{-1}\right)$}

The available phosphorus in surface layers ( 0 $25 \mathrm{~cm}$ ) of high, mid and low altitude soils ranged from32.7 to $41.6,33.1$ to 40.7 and 21.0 to $39.4 \mathrm{Kg}$ per hectare with mean value of $36.8,36.0$ and $29.8 \mathrm{Kg}$ per hectare, respectively, however, it varied from 25.5 to $37.1,22.8$ to 36.7 and $19.71-28.6 \mathrm{Kg}$ per hectare with mean value of $30.7,29.7$ and
$24.1 \mathrm{Kg}$ per hectare in subsurface layers (25$50 \mathrm{~cm}$ ) of high, mid and low altitude soils, respectively (Table 8). The available phosphorus status of pear orchard soils under study was found medium to high and it exhibited decreasing trend with an increase in soil depth. The available phosphorus was high in high altitude soils followed by mid altitude and low altitude soils, respectively. The available phosphorus in low altitude soils varied significantly with that of mid and high altitude soils, while as, its difference between mid and high altitude soils was at par statistically, which could be attributed to favourable soil reaction and high organic matter content leading to formation of organophosphate complexes and coating of iron and aluminum particles by humus. This is supported by the research work of Gupta $e t$ al., (1990) and Wani (2001).

\section{Potassium $\left(\mathrm{Kg} / \mathrm{ha}^{-1}\right)$}

In surface layers $(0-25 \mathrm{~cm})$ of high, mid and low altitude pear orchard soils of Kashmir, the available potassium varied from 356.1 to $474.8,331.5$ to 465.9 and 318.0 to $470.0 \mathrm{Kg}$ per hectare with mean value of $416.9,386.5$ and $401.6 \mathrm{Kg}$ per hectare, respectively, whereas, in subsurface layers $(25-50 \mathrm{~cm})$ it ranged from 277.7-407.6, 188.1-383.0 and $188.1-277.7 \mathrm{Kg}$ per hectare with mean value of $352.64,288.43$ and $235.84 \mathrm{Kg}$ per hectare, respectively (Table 9). The available potassium in high altitude soils differed significantly with that of mid altitude and low altitude soils, whereas, its difference between mid and low altitude soils was at par statically. The available potassium status of soils under study was high and it did not show any definite trend in its depth-wise distribution, which could be due to presence of high clay content and illitic nature of these clays. This is supported by the findings of Talib (1984) and Najar (2002). 
From the study it was concluded that the soils in general were medium to moderately fine textured with clay loam to silt clay loam as dominant texture in surface and subsurface layers. The soils were slightly acidic to slightly alkaline in reaction and $\mathrm{pH}$ exhibited an increasing trend with soil depth with lowest in surface soils of high altitude. The electrical conductivity was normal in surface and sub surface soils, respectively, with a decreasing trend with an increase in soil depth. The calcium carbonate was found to be higher in mid altitude soils indicating their calcareous nature. The organic carbon content was medium to high in soils under study in surface layers and decreased gradually with an increase in soil depth. The $\mathrm{pH}, \mathrm{EC}, \mathrm{CaCo}_{3}$ and $\mathrm{OC}$ exhibited significant differences with altitude.

The available nitrogen status of soils was low to medium. Available nitrogen showed a decreasing trend with soil depth. The available phosphorus exhibited a decreasing trend with an increase in soil depth and its status was found medium to high. The soils under study were high in available potassium without any definite trend in its vertical distribution. Thus, the available phosphorus and available potassium are by and large medium to high in pear orchard soils except nitrogen which is low in low altitude soils. The study revealed that altitude bears effect on the available primary nutrients and physico-chemical properties of soils which may be due to climatic factors and mineralogical composition of soils.

\section{References}

Anonymous,2007. Area and production of horticultural crops in Jammu \& Kashmir State. Digest of Statistics 2006-2007, Directorate of Economics and Statistics, J\&K Government 33(07): 46-52.
Balanagoudar, S.R. and Satyanarayana, T. 1990. Correlations of different forms of sulphur with soil properties and with organic carbon and nitrogen in some Vertisols and Alfisols. Journal of the Indian Society of Soil Science 38:641645.

Farida,A. 2005. Studies on relationship between fruit yield and quality with soil and leaf nutrient content in apple orchards of Zangier block of district Baramulla, Kashmir. Ph.D. thesis submitted to Sher-e-Kashmir University of Agricultural Sciences \& Technology of Kashmir, Shalimar, Srinagar,pp117.

Gupta,R.D.,Khanna,Y.P. and Singh,M. 1990. Identification of clay minerals in some cultivated and uncultivated soils of Rajouri and Poonch districts of Jammu and Kashmir.Clay Research 9: 19-24.

Haverkort,A.J. 1987. Climate and potato crop growth interactions in Africa's continential divide region.Acta Horticulturae 214; 137-147.

Jackson,M.L. 1973. Soil Chemical Analysis. $2^{\text {nd }}$ edition. Printice Hall of India,New Delhi,pp498.

Kaistha,B.P.,Sood,R.D. and Kanwar,B.S. 1990. Distribution of nitrogen in some forest soil profiles of north Western Himalayan region. Journal of the Indian Society of Soil Science 38: 15-20.

Kher,D. and Singh,N. 1993. Forms of sulphur in mustard growing soils of north Kashmir. Journal of the Indian Society of Soil Science 40: 164-165.

Lahiri,T. and Chakravarti,S.K. 1989. Characterization of some soils of Sikkim at various altitudes. Journal of the Indian Society of Soil Science 37: 451-454.

Mandal,A.K., Nath, S., Gupta,S.K. and Banerjee, S.K. 1990. Characterization and nutritional status of soils of middle and upper hill forest of the eastern 
Himalayas.Journal of the Indian Society of Soil Science 38: 100-106.

Minhas,R.S. and Bora,N.C. 1982. Distribution of organic carbon and the forms of nitrogen in topographic sequence of soil.Journal of the Indian Society of Soil Science 30: 135-139.

Mushki,G.M. 1994. Studies on apple orchard soils of kashmir.M.Sc. thesis submitted to Sher-e-Kashmir University of Agricultural Sciences \& Technology of Kashmir,Shalimar, Srinagar pp144.

Najar, G.R. 2002. Studies on pedogenesis and nutrient indexing of apple(Red Delicious) growing soils of Kashmir. $\mathrm{Ph} . \mathrm{D}$. thesis submitted to Sher-eKashmir University of Agricultural Sciences \& Technology of Kashmir, Shalimar, Srinagar pp204.

Najar,G.R., Rahman,F.H. and Farida,A. 2006. Effect of altitude on distribution and forms of nitrogen in some apple growing soils of Kashmir.Environment and Ecology 24S(2): 433-436.

Olsen,S.R., Cole,C.V.,Watanabe,F.S. and Pean,L.A. 1954. Estimation of available phosphorus by extracting with sodium bicarbonate. U.S.D.A. Circular No.939.

Piper,C.S. 1966. Soil and Plant Analysis. $5^{\text {th }}$ edition. Hans Publisher, Bombay pp 464.

Sharma,B.D., Mukhopadhyay,S.S. and Arora,H. 2005. Total and DTPA extractable micro-nutrients in relation to pedogenesis in some Alfisols of Punjab, India. Soil Science 170(7): 559-572.

Subbiah,B.V. and Asija,G.L. 1956. A rapid procedure for estimation of available nitrogen in soils.Current Science 25: 259-260.

Talib, A.R. 1984. Studies on the pedogenesis and potassium supplying capacity of the benchmark soils of Kashmir.Ph.D. thesis submitted to Himachal Pradesh Krishi Vishwavidyalaya, Palampur,H.P. pp 144.

Walkley,A. and Black,I.A. 1934. An examination of the method for determining soil organic matter and a proposed modification of chromic acid titration.Soil Science 37(1-6): 29-39.

\section{How to cite this article:}

Dar, M.A., Rehana Rasool, Masrat Maqbool, J.A. Wani, M.Y. Bhat and Shazia Ramzan. 2017. Depth Wise Distribution of Primary Nutrients in Pear Orchard Soils of Kashmir. Int.J.Curr.Microbiol.App.Sci. 6(6): 2526-2539. doi: https://doi.org/10.20546/ijcmas.2017.606.301 